\title{
Improving the Interplay between Usability Evaluation and User Interface Design
}

\author{
Kasper Hornbæk \\ Department of Computing \\ University of Copenhagen \\ Universitetsparken 1, DK-2100 Copenhagen \\ Denmark \\ kash@diku.dk
}

\author{
Jan Stage \\ Department of Computer Science \\ Aalborg University \\ Fredrik Bajers Vej 7, DK-9220 Aalborg \\ Denmark \\ jans@cs.aau.dk
}

\begin{abstract}
This paper provides an overview of a full-day workshop that was held on October 232004 in connection with the Third Nordic Conference on Human Computer Interaction (Nordichi 2004). The proceedings from the workshop are available from http://www.cs.aau.dk/ jans/events.html.

The ideas and theme of the workshop are motivated and an outline of the contents of the papers that were presented in the workshop is given. In addition we summarize some challenges to the interplay between usability evaluation and user interface design agreed upon at the workshop, as well as some solutions that were debated.
\end{abstract}

\section{Introduction}

Software development is highly challenging. Despite many significant successes, several software development projects fail completely or produce software with serious limitations, including (1) lack of usefulness, i.e. the system does not adequately support the core tasks of the user, (2) unsuitable designs of user interactions and interfaces, and (3) lack of productivity gains or even reduced productivity despite heavy investments in information technology (Gould \& Lewis 1985, Strassman 1985, Brooks 1987, Matthiasen \& Stage 1992, Nielsen 1993, Attewell 1994, Landauer 1995).

Broadly speaking, two approaches have been taken to address these limitations. The first approach is to employ evaluation activities in a software development project in order to determine and improve the usability of the software, i.e. the effectiveness, efficiency and satisfaction with which users achieve their goals (ISO 1998, Frøkjær et al. 2000). To help software developers' work with usability within this approach, more than 15 years of research in HCI has created and compared techniques for evaluating usability (Lewis 1982; Nielsen \& Mack 1994).

The second approach is based on the significant advances in techniques and methodologies for user interface design that have been achieved in the last decades. In particular, researchers in user interface design have worked on improving the usefulness of information technology by focusing on a deeper understanding on how to extract and understand user needs. Their results today constitute the areas of participatory design and user-centered design (e.g. Greenbaum \& Kyng 1991, Beyer \& Holtzblatt 1998, Bødker, Kensing \& Simonsen 2004).

However, the interplay between these two approaches, and between the activities they advocate to be undertaken in software development, have been limited. Integrating usability evaluation at relevant points in user interface design with successful and tothe-point results has proved difficult. In addition, research in $\mathrm{HCI}$ and software design has been done mainly independently of each other with no in substantial exchange of results and sparse efforts to combine the techniques of the two approaches. Larry Constantine, a prominent software development researcher, and his colleagues express it this way: "Integrating usability into the software development process is not easy or obvious" (Juristo et al. 2001, p. 21).

\section{Idea of the Workshop}

The idea of the workshop was to inquire in more detail into the interplay between design and usability evaluation. Software development is the overall process that we focus on. Within this process there is a multitude of different activities. Two key activities are user interface design and usability evaluation, see figure 1. The purpose of usability evaluation is to assess the usability of user interface designs. This assessment is based on different design products, e.g. mockups, prototypes, incomplete versions of the final system or even the final system itself. In the usability evaluation activity these design products are assessed and the results are fed back into the user interface design activity. The results can also take a variety of forms, e.g. the traditional usability report with problems lists, video clips, redesign proposals or verbal briefings.

This description represents the ideal case. In reality, the interplay is more complicated. The design products may be unusable as a basis for evaluation and they are available too late in the development process. The evaluation process often takes too long, and the results seem to have a very limited effect on the design process.

The literature on HCI does not provide help on this problem. The $\mathrm{HCI}$ field includes a rich variety of techniques for either usability

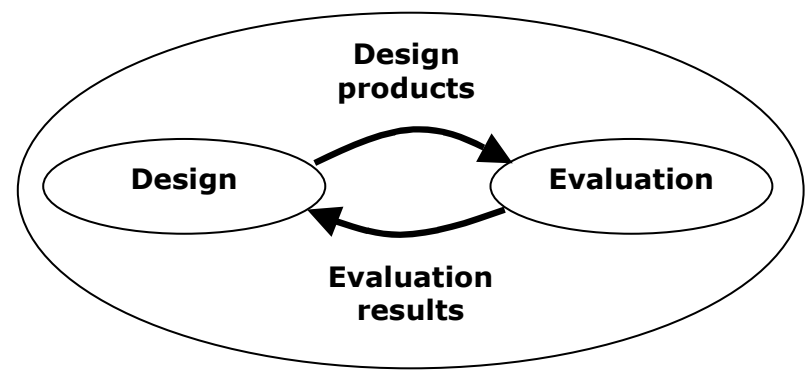

Figure 1. The interplay between user interface design and usability evaluation as key activities in software development 
evaluation or user interface design. But there are very few methodological guidelines for the interplay between these key activities. In addition, there are no systematic surveys of research in this area.

\section{Goal of the Workshop}

The goal of the workshop was to determine state-of-the-art in the interplay between usability evaluation and user interface design and to generate ideas for new and improved relations between these activities. The aim was to base the determination of the current state on empirical studies. Thus authors were asked to employ empirical studies as a basis for presentations of new ideas on how to improve the interplay. Within this focus, the following topics of discussion were suggested:

- Which products of user interface design are useful as the basis for usability evaluations?

- How do the specific products from user interface design influence the techniques that are relevant for the usability evaluation?

- In which forms are the results of usability evaluations supplied back into user interface design?

- What are the characteristics of usability evaluation results that are needed in user interface design?

- Do existing evaluation methods deliver the results that are needed in user interface design?

- How can usability evaluation be integrated more directly in user interface design?

- How can usability evaluation methods be applied in emerging techniques for user interface design?

\section{Overview of Papers}

Ten papers were accepted for the workshop. They are divided into the following four groups:
A. Case studies of design and evaluation
B. User centered design (UCD)
C. Impact on Software Development
D. Reframing the Problem

Group A includes three papers that present results from empirical studies of the interplay between user interface design and usability evaluation. Kadyte and Tétard describes how usability evaluation was conducted and which usability testing techniques that were employed in the development of a mobile system. The usability evaluation helped the designers get a better understanding of the consequences of choosing different design options.

Murphy et al. reports from a project where usability evaluation was outsourced to an external evaluation organization. It is described how the evaluation process was structured, and the usefulness of different kinds of feedback from evaluation to design is discussed.

Paay and Kjeldskov presents the design of a prototype of an indexical context-aware mobile system. It is described how an understanding of the context of use is useful for planning usability evaluations.
Group B includes two papers that inquire into the extent to which the user centered design approach provides a way of handling the interplay between user interface design and usability evaluation. Venturi focuses on the extent to which user centered design techniques are used in the software industry, particularly in combination with the Rational Unified Process (RUP). Two patterns of integration are described and the challenges of integration are discussed.

Lárusdóttir presents a research plan for comparing the waterfall model with a user centred design approach. The research is based on student projects, and guidelines for these are also discussed.

Group $\mathrm{C}$ includes three papers that focus on key aspects of usability evaluation. Frøkjær and Hornbæk deals with feedback from evaluation to design. They have conducted interviews with designers in order to determine elements of feedback that are particularly valuable. They conclude that redesign proposals as opposed to mere problem lists are very valuable for software designers.

Skov and Stage inquire into the challenges of integrating usability evaluation into the design process by having designers conduct usability evaluations. A simple introduction to usability engineering is outlined and the results from teaching this to novice evaluators are presented. It is concluded that the novices became capable in some areas of usability engineering, but in others they still lacked competence.

Law deals with effectiveness of usability evaluation methods. Based on data from usability evaluations, it is discussed to what extent the problems identified induce fixing. It is also discussed more generally what effectiveness of a usability evaluation method is.

Group D includes two papers that provide a reframing of the topic. Hvannberg focuses on the relation between elicitation and design and between design and evaluation. The discussion is based on two case studies. It is suggested that design and evaluation are run concurrently in the development process with two related models as repositories.

Cockton argues that user interface design and usability evaluation both have to be placed within a value-centred framework. Usability evaluation deals with interaction, not designs. A valuecentred approach is motivated and outlined; with that approach most of the questions raised in the call for workshop papers are reframed or rejected.

\section{Challenges discussed at the workshop}

To us, five challenges discussed at the workshop reading the interplay between evaluation and design stand out. They concern (1) the form and content of feedback from usability evaluation to user interface design; (2) achieving an early interplay between evaluation and design; (3) improving commitment towards and understanding of HCI and usability evaluation; (4) methodological problems in the research on usability evaluation and user interface design; and (5) challenges imposed by changing contexts of software development.

First, an important challenge concerns the form of feedback given from evaluation to design. Typically, user interface designers receive as feedback a report, listing usability problems with their design. However, several participants at the workshop argued that this form of output is problematic because the problems in the 
report are often very short, too numerous, detached from the context in which they arose, and hard to understand. In addition, it is doubtful whether listing of problems are a key concern in actual software development. Previous research also suggests that not all problems raised in such reports are equally important; some problems may lead designers to waste time, should they try to correct them. Yet, research examining alternative forms of output from usability evaluation is rare.

Second, achieving early interplay between evaluation and design was identified as a key challenge. In particular participants agree that rescue HCI, that is late and cosmetic impact of evaluation on design, was unsatisfactory. Rescue HCI, however, seems to be happening a lot in software development. While this role of HCI in design to some extent may be the fault of HCI professionals themselves, the challenge to have early and value adding influence on the design of products nevertheless remains. The key here is to make usability evaluation be a part in shaping what gets designed.

Third, getting an understanding for how HCI may contribute to the software development have proven to be challenging; getting commitment to early and continuous focus on usability evaluation is even harder. These challenges include managing expectations of software designers, and being clear about what (and what not) HCI can do. Improving the relation between management and HCI professionals in particular, seems important: reward structures and top-level support on HCI are rarely in place. Several participants argued that too often management or designers hold unrealistic expectations, causing a sure-loss situation for usability evaluation and its interplay with software design.

Fourth, a number of methodological challenges were discussed, including the core issue of how to assess the ability of usability evaluation methods to impact user interface design. In particular, several participants questioned the reliance upon think aloud testing as a gold standard against which to assess alternative usability evaluation techniques. Another issue concerned how to ensure the validity of the usability issues identified with a product - while much research has produced usability evaluation techniques that can find many usability problems, little research have documented that those problems are real, let alone have useful impact on user interface design. Finally, many techniques and measures of HCI emphasize task-related performance measures, for example task completion times or accuracy. As products and services that we want to evaluate are increasingly dealing with experiences, games, and long-term interaction, we need to find better measures of subjective experience in order to, for example, make these criteria of iteration. However, especially linking those measures to design proposals seems hard.

Fifth, recent changes in software development contexts were discussed - for example diminishing time to market, faster development cycles and new devices. These challenges appear in practice to impose many constraints on the interplay between usability evaluation and user interface design. For example, the faster development cycles mean that less time is available for the actual evaluation, quicker analysis is needed, and more clear-cut advice is needed. Usability evaluation techniques and tools for these contexts are lacking.

\section{Solutions Discussed at the Workshop}

While challenges were numerous and easily describable, solutions were sketchier. Below we describe some of them.

One recurring suggestion was for more empirical studies of industrial scale design projects, thereby raising our understanding of the interplay between design and evaluation as it unfolds in practical projects. The focus of such studies could include how developers assess and chose to correct usability problems, the impact of various form of problem descriptions, and the evaluation of different representations of design, say use cases compared to paper prototypes. Such studies could also serve as exemplar case studies to be used in establishing realistic expectations of how usability evaluation and HCI could impact design. Initial explorations in this direction were presented by Lárusdóttir, Frøkjær and Hornbæk, and Law.

Another key idea was to strengthen the coupling of evaluation and goals/values of the design. All too often, evaluation is done with a too shallow understanding of the goals and values to be embodied by the design; evaluation also is done too late to matter. Several position papers presented ideas on how to feed information from design activities into the evaluation activities, for example through value statements and testable design rationales.

As evident from the section on challenges above, much more research is needed on the various form of feedback in which the results of usability evaluation is presented to developers. Such forms include redesign proposals, video highlights, and workshops. All of these have been at least initially explored with interesting results; however, studies examining the impact and persuasiveness of various forms of feedback are needed. In particular, the needs and wants of stakeholders in the design process should be carefully considered in relation to finding suitable and persuasive forms of feedback.

A supplement to the above ideas is to improve evaluators' skills. Little research has aimed at improving in concert the finding, analysis, filtering, and reporting of problems. The basic idea presented by Skov and Stage was to circumvent the gap between evaluators and developers by teaching basic evaluation skills to developers.

While the focus of the workshop was on empirical studies, the position papers made it plain that further work is needed before any clear solutions to improving the interplay between evaluation and design are reached.

\section{References}

Attewell, P. (1994), Information technology and the productivity paradox. In D.H. Harris (eds), "Organizational Linkages: Understanding the Productivity Paradox". Washington, DC: National Academy Press.

Beyer, H. \& Holtzblatt, K. (1998), Contextual design, Morgan Kaufman Publishers

Brooks, Jr., F. P. 1987. No Silver Bullet: Essence and Accidents of Software Engineering, IEEE Computer, 20, 10-19.

Bødker, K., F. Kensing, and J. Simonsen (2004): Participatory IT Design. Designing for Business and Workplace Realities.

Frøkjær, E., Hertzum, M., \& Hornbæk, K. (2000), "Measuring usability: are effectiveness, efficiency, and satisfaction really 
correlated?", Proceedings of CHI 2000, 345-352 ,The Hague Netherlands:ACM Press.

Gould, J. D. \& Lewis, C. (1985), "Design for usability: Key principles and what designers think", Communications of the ACM, 28(3), 300-311.

Greenbaum, J. \& Kyng, M. (eds.) (1991), Design at work: cooperative design of computer systems, Lawrence Erlbaum Ass.

ISO (1998), "Ergonomic requirements for office work with visual display terminals (VDTs)-Part 11: Guidance on Usability".

Juristo, N., Windl, H., \& Constantine, L. (2001), "Introducing usability", IEEE Software, 20-21.
Landauer, T. K. (1995), The trouble with computers: usefulness, usability, and productivity, MIT Press.

Lewis, C. (1982), "Using the "thinking-aloud" method in cognitive interface design", Research Report RC9265.

Mathiassen, L. and Stage, J. (1992) The Principle of Limited Reduction in Software Design. Information Technology \& People, 6(2-3):171-185.

Nielsen, J. \& Mack, R. L. (1994), Usability Inspection Methods, Wiley and Sons Inc.

Strassman, P. A. (1985), Information Payoff: The Transformation of Work in the Electronic Age, New Canaan, CT: The Information Economic Press. 